\title{
Objetos de Aprendizagem e Pensamento Computacional: um levantamento acerca do ensino de computabilidade
}

\author{
Gustavo Silva Semaan ${ }^{1}$, Glaucia Ribeiro Gonzaga ${ }^{1}$, Debora Alvernaz Corrêa ${ }^{2}$, \\ Gabriel Marçal Araújo ${ }^{1}$, José André de Moura Brito ${ }^{3}$, Edelberto Franco Silva ${ }^{4}$ \\ ${ }^{1}$ Instituto do Noroeste Fluminense de Educação Superior \\ Universidade Federal Fluminense (UFF) - Santo Antônio de Pádua, RJ, Brasil \\ ${ }^{2}$ Centro Universitário Descomplica UniAmérica - Rio de Janeiro, RJ, Brasil \\ ${ }^{3}$ Escola Nacional de Ciências Estatísticas (ENCE) - Rio de Janeiro, RJ, Brasil \\ ${ }^{4}$ Departamento de Ciência da Computação - Universidade Federal de Juiz de Fora \\ (UFJF) - Juiz de Fora, MG, Brasil \\ gustavosemaan@id.uff.br, glauciargonzaga@gmail.com, elberto@ice.ufjf.br
}

Resumo. O Pensamento Computacional já é considerado uma das habilidades intelectuais básicas do humano, sendo comparado à leitura e capacidade de realizar operações aritméticas, e a influência do "mundo digital" no cotidiano já está presente na Lei de Diretrizes e Bases da Educação Nacional (LDB). Nesse sentido, o uso de ferramentas educacionais, como Objetos de Aprendizagem (OA), que promovam desenvolvimento cognitivo necessário à construção desse conhecimento e suas associações, são de suma importância. O presente trabalho apresenta uma revisão da literatura sobre OA relacionados à Computabilidade, tendo por base a análise de mais de 15,5 mil artigos de acervos importantes relacionados. Nesta revisão foram identificadas lacunas no cenário nacional quanto à temática, em especial para a Educação Básica.

Abstract. Computational Thinking is already considered one of the primary intellectual skills of a human being, compared to reading and performing arithmetic operations; besides that, the influence of the "digital world" in daily life is already present in the Law on Brazilian Education Guidelines and Bases. Thus, educational tools (such as Learning Objects - LO) that promote the cognitive development necessary to build this knowledge and its associations are of great importance. The present work introduces a literature review on $L O$ related to Computability and analyzes more than 15,500 articles. In this review, gaps were identified in the national scenario, especially regarding the theme for Basic Education.

\section{Introdução}

O impacto da Tecnologia da Informação (TI) nas mais diversas áreas do conhecimento acadêmico e no ambiente empresarial é cada vez maior e mais profundo. Existem diversos problemas que, sem os instrumentos da TI não seriam resolvidos ou seriam impraticáveis a obtenção de soluções devido às restrições de tempo e o custo financeiro demandados. Tais problemas que admitem uma implementação nos recursos atuais disponíveis são 
ditos computáveis, quais sejam: computadores pessoais, "supercomputadores", ou até mesmo nas "nuvens" (do inglês Cloud Computing).

Porém, inerente ao assunto computabilidade, há o estudo de complexidades, em que devem ser analisados os requisitos de tempo e espaço de memória necessários à execução de um dado programa, que foi codificado com base em instruções existentes em um roteiro, denominado algoritmo. Ademais, muitos problemas são classificados como difíceis, uma vez que sua resolução é impraticável, a despeito os instrumentos de TI mais sofisticados existentes (BRACKMANN, 2017).

A revisão da literatura realizada nesse estudo buscou trabalhos que relacionam Pensamento Computacional (PC, do inglês Computational Thinking), Objetos de Aprendizagem (OA, do inglês Learning Objects), Informática na Educação, e termos associados à temática Computabilidade, em especial sobre Intratabilidade e Otimização, que são caracterizados pelo grande interesse e demanda em áreas como a Computação e as Engenharias no cenário nacional.

Para a revisão da literatura foram analisados, essencialmente, quatro importantes acervos: RENOTE ${ }^{1}, \mathrm{RBIE}^{2}, \mathrm{SBIE}^{3}$ e $\mathrm{SIMPEP}^{4}$, e que contemplam um total de 15.561 trabalhos publicados no período entre 1997 e 2020. Esses acervos têm grande relevância pela alta qualidade de seus trabalhos e forte aderência ao contexto da pesquisa.

O objetivo principal desse trabalho é buscar conteúdos relacionados com OA e PC em relação à temática Computabilidade. Os referidos instrumentos de ensino podem ser utilizados desde a primeira infância, como apontado por Silva (2017) em sua pesquisa envolvendo o uso das tecnologias digitais com crianças de 7 meses a 7 anos, nos ensinos fundamental e médio. No ensino superior, a ausência de estudos nesse sentido foi reportada por Zanetti, Borges e Ricarte (2016). Por fim, destaca-se que os assuntos relacionados a Problemas Computacionais, como os da área Otimização, são amplos e podem ser utilizados nas mais diversas aplicações e situações do mundo real, inclusive nas áreas de Ensino e Educação. Trata-se de conteúdos utilizados no cotidiano, nas mais diversas situações, desde a organização de horários de atividades, arrumação de malas antes de viagens; até na escolha do caminho entre a casa e o trabalho (escola).

O artigo segue organizado em 5 seções, a partir desta introdução. A seção 2 apresenta os Trabalhos Relacionados. Na seção 3 são apresentados conceitos e definições em relação ao PC e aos OA. A seção 4 discorre os tipos de Problemas Computacionais em uma abordagem ilustrativa e de simples entendimento. Por fim, na seção 5 são feitas as considerações finais e propostas para trabalhos futuros.

\section{Trabalhos Relacionados}

A revisão da literatura realizada nesse estudo buscou por trabalhos que relacionam assuntos acerca da temática Computabilidade e Instrumentos e Técnicas da Informática na Educação, em busca de contribuições efetivas concernentes ao desenvolvimento do PC. Para isso, foram analisados, essencialmente, mas não de maneira restrita, os repositórios associados aos acervos citados na seção 1 (Introdução).

\footnotetext{
${ }^{1}$ Revista Novas Tecnologias na Educação (17 edições, 40 números e 1.866 artigos entre 2003 e 2019).

${ }^{2}$ Revista Brasileira de Informática na Educação (61 edições e 484 artigos entre 1997 e 2020 ).

${ }^{3}$ Simpósio Brasileiro de Informática na Educação (18 edições e 2.147 artigos entre 2001 e 2018).

${ }^{4}$ Simpósio de Engenharia de Produção (21 edições e 11.064 artigos entre 1998 e 2018).
} 
A Tabela 1 apresenta, de maneira resumida, os quantitativos de trabalhos localizados por busca em cada acervo. Observa-se que termos relacionados à Informática na Educação possuem destaque na RENOTE, RBIE e SBIE, como o esperado. Porém, os termos relacionados à temática Computabilidade retornaram poucas ocorrências nesses acervos. De maneira semelhante, mas em sentido oposto, a busca no acervo do SIMPEP resultou em apenas 2 artigos em relação a Objetos de Aprendizagem, e nenhum sobre TICs ou sobre Pensamento Computacional, mas ao buscar pelos termos Otimização ou Pesquisa Operacional foram catalogados mais de 170 artigos. Trata-se de áreas em destaque no simpósio, porém, para efetivamente localizar artigos com o escopo esperado nesse trabalho, foi necessário filtrar pelas áreas relacionadas à Educação: (i) Educação em Engenharia de Produção e (ii) Educação, Ética e Formação da Pessoa. Tal filtro reduz significativamente o quantitativo de artigos, para apenas quatro unidades.

Entre os resultados obtidos na busca por Pensamento Computacional, os trabalhos de Zanetti, Borges e Ricarte (2016) e Carvalho et. al (2017) foram publicados recentemente, e apresentam Revisões Sistemáticas da Literatura (RSLs) de grande interesse nessa pesquisa, embora não tratem especificamente a Computabilidade. Bombasar et. al (2015) em sua RSL destacam a ausência de ferramentas inspiradas nas teorias e formalismos desenvolvidos por Alan Turing (considerado o pai da computação moderna), e sugerem o desenvolvimento de OA inspirados no funcionamento de uma Máquina de Turing (MT) para conduzir a uma forma de pensar e programar ainda pouco explorada, presente na descrição do PC apresentada por Wing (2006).

Tabela 1: Revisão sistemática da literatura: termos de busca por acervo.

\begin{tabular}{l|r|r|r|r}
\hline Termos de busca & RENOTE & RBIE & SBIE & SIMPEP \\
\hline Objetos de Aprendizagem & 147 & 40 & 179 & 2 \\
\hline TICs (Tecnologias da informação e comunicação) & 57 & 7 & 21 & 0 \\
\hline Pensamento Computacional & 16 & 9 & 49 & 0 \\
\hline Otimização & 7 & 5 & 12 & $150(2 *)$ \\
\hline Pesquisa Operacional & 1 & 2 & 12 & $26(2 *)$ \\
\hline Problemas Computacionais & 0 & 1 & 0 & 0 \\
\hline Teoria da Computação & 0 & 3 & 1 & 0 \\
\hline Alan Turing *Ao filtrar por artigos das áreas relacionadas à Educação. & 0 & 0 & 1 & 0 \\
\hline Intratabilidade & 0 & 0 & 0 & 0 \\
\hline \multicolumn{4}{|r|}{}
\end{tabular}

Com base na revisão da literatura do acervo da RENOTE, após a busca e análise dos resultados, foram destacados dois trabalhos de maior interesse no contexto da pesquisa. Em Cordenonsi, Muller e Bastos (2006) é citado um "vácuo tecnológico e pedagógico" a ser preenchido no ensino de (meta)heurísticas (SEMAAN et al., 2020), assunto de grande importância para resolução de problemas intratáveis e de otimização, e propõe uma nova metodologia com base na Educação Dialógico-Problematizadora, com o intuito de "consolidar uma construção participativa dos alunos". Já Santos, Camponogara e Bernardon (2016) destacam a necessidade de inovar em métodos de ensino, e através da Aprendizagem Baseada em Problema (do inglês Problem Based Learning) propuseram um painel didático iterativo e interativo a partir de equipamentos de baixo custo, com o objetivo de apoiar o ensino operacional de sistemas de distribuição de energia elétrica.

Em relação às buscas nos anais do SIMPEP, dois trabalhos que tratam de OA têm objetivos que não estão no escopo deste trabalho. Apesar de existirem trabalhos mais aderentes ao objetivo da presente pesquisa, não contemplam PC por meio de OA. Além 
disso, nenhum trabalho relacionado teve como foco na primeira infância ou mesmo nos alunos da educação básica (ensino fundamental e/ou ensino médio), em que abordagens específicas podem ser consideradas para uma introdução a problemas computacionais.

\section{Pensamento Computacional e Objetos de Aprendizagem}

Os conhecimentos adquiridos com os avanços da TI podem ajudar a melhorar a vida das pessoas, quando utilizados para a criação de soluções inovadoras ou mesmo em seu cotidiano. Essas soluções são absorvidas e consumidas naturalmente, e muitas vezes não se percebe o quanto são poderosas e influenciadoras. Por exemplo, aplicativos (Apps) para envio de mensagens para celulares suportam, além de textos, o envio de sons, fotos, vídeos, sendo também capazes de fazer ligações e chamadas de vídeo.

Com uso frequente de alguns apps, em especial aqueles relacionados à comunicação, pode-se questionar se as empresas de telefonia são, na verdade, provedores de internet, e afastam-se, cada vez mais, de seu produto principal inicial. Os smartphones tornaram-se computadores de bolso que, com acesso a redes sem fio locais como em sua casa, independem de empresas de telefonia móvel para seu "pleno" funcionamento, com base nos recursos e aplicativos utilizados com maior frequência.

Os conhecimentos em TI são de grande importância para a vida na sociedade contemporânea (BRACKMANN, 2017). Conforme Wing (2006), o uso do PC deve ser equiparado às habilidades básicas de leitura, escrita e aritmética. De modo objetivo, ele emprega técnicas e conceitos da Ciência da Computação para resolver problemas do cotidiano (BOMBASAR et al., 2015). Essa grande influência do "mundo digital" já está presente na Lei de Diretrizes e Bases (LDB), em que é objetivo da formação básica do cidadão "a compreensão do ambiente natural e social, do sistema político, da tecnologia, das artes e dos valores em que se fundamenta a sociedade" (LDB, 2021).

A Royal Society $(2012$, p.29) define o PC como "o processo de reconhecer os aspectos computacionais no mundo que nos rodeia, e aplicar ferramentas e técnicas da Ciência da Computação para compreender e raciocinar sobre os sistemas e processos naturais e artificiais". Assim, ela está em conformidade com a LDB, que cita a compreensão da tecnologia como parte da formação básica.

As definições normativas do PC são muito úteis para o entendimento de sua essência, porém não especificam os tipos de conteúdo adequados para os processos de ensino e de aprendizagem, que possuem críticas por serem considerados vagos. De fato, deve-se questionar se tais críticas são adequadas, visto a liberdade de criação que deve existir para a promoção de uma ideia e adaptação ao contexto conforme a demanda. Por outro lado, existe uma preocupação em se adotar o PC em substituição a um ensino mais abrangente de ciência, além do receio de que a abordagem estimule uma maneira de pensar menos abrangente, limitada a problemas a serem resolvidos, ignorando ou não destacando impactos sociais, éticos e ambientais envolvidos (EASTERBROOK, 2014).

A definição operacional oferecida pela CSTA (Computer Science Teachers Association) em conjunto com a ISTE (International Society for Technology in Education), torna-se mais adequada para pesquisas envolvendo práticas de ensino e de aprendizagem, em que o PC diz respeito a um processo de resolução de problemas que inclui características como: (i) formulação de problemas computáveis; (ii) organização, análise e representação de dados através de modelos e simulações; e (iii) implementação 
de soluções visando a otimização de passos e recursos, bem como a generalização de soluções para outros problemas.

Em um trabalho de Syslo e Kwiatkowska (2014) é discutido como a Ciência da Computação pode contribuir no ensino de matemática, e o PC é abordado por dois caminhos, quais sejam: (i) com o intuito estender e enriquecer o ensino de tópicos tradicionais da matemática, através da Computação como disciplina e dos computadores e softwares como ferramentas de apoio; (ii) em contribuições na construção do conhecimento por meio de soluções para problemas do mundo real, apresentados como problemas computacionais (tratáveis e intratáveis) (SEMAAN et al., 2020).

Em relação aos OA, trata-se de uma abordagem recente e sem um consenso universal quanto à sua definição formal. Entretanto, é indispensável que eles sejam pensados de maneira a possibilitar sua reutilização enquanto conteúdo educacional, bem como seu funcionamento como mediador do conhecimento (GALAFASSI, GLUZ, GALAFASSI, 2014; BRAGA, MENEZES, 2014). Por exemplo, Braga e Menezes (2014, p.13) definem os OA como "componentes ou unidades digitais, catalogados $e$ disponibilizados em repositórios na Internet para serem reutilizados para o ensino".

Conforme o IEEE Learning Technology Standards Committee (IEEE, 2002), um OA trata-se "Qualquer entidade, digital ou não, que pode ser usada, reutilizada ou referenciada durante o aprendizado apoiado pela tecnologia". Em direção semelhante a essa definição, os autores do presente trabalho consideram que abordagens não digitais podem ser caracterizadas como OA, como, por exemplo, as atividades Computação Desplugada (CSUNPLUGGED, 2021; CARVALHO et al., 2017).

Destaca-se que a adição de tais OA não digitais em repositórios na internet, mesmo que seja através de tutoriais e/ou vídeos explicativos, contribuem de maneira efetiva com a popularização e democratização do conhecimento, além de favorecer sua efetiva reutilização. Nesse sentido, Braga e Menezes (2014) apresentam uma relação com 15 importantes repositórios nacionais e internacionais de OA.

$\mathrm{Na}$ área da educação as inovações surgem com rapidez, aprimorando técnicas que visam facilitar os processos de ensino e aprendizagem. Grandes empresas e instituições de ensino consagradas apoiam o desenvolvimento de OA que, mediante o uso de abordagens lúdicas e dinâmicas, facilitam o processo de ensino de conceitos da Ciência da Computação. Tais ferramentas podem ser utilizadas em diferentes contextos e funcionam como mediadores do conhecimento. Elas estimulam e facilitam desde a interpretação à resolução de problemas, promovendo o PC (CARVALHO et al., 2017).

\section{Problemas Computacionais}

A presente seção aborda brevemente a temática computabilidade, e traz a distinção entre problemas considerados computáveis, passíveis de submissão a um instrumento de TI, e os considerados insolucionáveis, que podem não ter solução. De modo adicional, é importante destacar que um problema computável pode ser intratável conforme: (i) os dados de entrada; (ii) a maneira de resolvê-lo; (iii) os instrumentos de TI disponíveis para isso; (iv) ou mesmo uma combinação desses fatores. São os problemas classificados, de modo informal, como "dificeis".

Um problema computacional pode ser considerado tratável (ou fácil) se existir uma maneira de resolvê-lo com custo aceitável de tempo e de espaço (memória). 
Formalmente, trata-se da existência de um algoritmo polinomial (cujo número de operações é limitado por uma função polinomial) capaz de resolvê-lo. Já para os problemas intratáveis os recursos disponíveis podem ser insuficientes para sua resolução, em especial no tocante ao tempo de processamento e/ou espaço (memória). Por fím, existem os problemas insolúveis (ou indecidíveis), e pode não haver garantia de sua resolução por meio do uso de máquinas (TI em geral) (SIPSER, 2005).

Em um primeiro momento é importante diferenciar a complexidade de um problema e dos algoritmos para solucioná-lo. Para isto, basta pensar que, para um dado problema, uma maneira de resolvê-lo (um algoritmo, por exemplo) pode não ser a melhor (a mais eficiente ou a mais eficaz), que resulta na melhor resposta e que consumiu menos recursos (tempo e espaço/memória). Embora importante no contexto, a análise de complexidade de algoritmos não é o foco desse trabalho (SIPSER, 2005).

A frase "Computação não é mais sobre computadores do que Astronomia é sobre telescópios", atribuída ao pesquisador Edsger W. Dijkstra, diz muito sobre o contexto da presente pesquisa. De maneira complementar, "O propósito da computação é o insight, não os números" (HAMMING, 2012). Mesmo com toda a infraestrutura de TI, deve-se destacar a importância dos três estágios do PC, independente de uso de equipamentos tecnológicos: (i) Entendimento e formulação do problema (abstração); (ii) Expressão da solução (automação); (iii) Execução da solução e avaliação (análise).

Mesmo antes da introdução dos conceitos iniciais da TI e do PC, Polya (1945) propôs a adoção de um conjunto limitado de estratégias para a resolução de problemas, como: (i) Busca por analogia: verificar se algum problema semelhante já possui uma solução adequada, e se é possível adequá-la para a resolução do problema em questão; (ii) Indução: deve-se resolver alguns exemplos simples e, com base no conhecimento adquirido, formular uma solução inteligente; (iii) Divisão: decompor um problema em subproblemas menores, resolvê-los e agrupá-los para a formação de uma solução unificada. De fato, tais estratégias são fortemente consideradas, talvez como um arcabouço da Ciência da Computação.

Embora a interação com novas tecnologias seja algo rotineiro entre alguns jovens e crianças, acredita-se que eles têm pouca experiência e grandes limitações para criá-las. Conforme (BRACKMANN, 2017, p.19), "é quase como se conseguissem ler, mas não conseguissem escrever com as novas tecnologias". Assim, um elo importante dos conceitos de $\mathrm{PC}$ em relação às habilidades básicas de leitura, escrita e aritmética com a programação é que, "no momento que os estudantes aprendem a programar, estão também programando para aprender" (BRACKMANN, 2017, p.20).

\section{Conclusões e Trabalhos Futuros}

O objetivo principal do trabalho foi apresentar uma revisão da literatura sobre Objetos de Aprendizagem relacionados com tópicos da Teoria da Computação. Para isso, foram analisados essencialmente, mas não de maneira restrita, quatro importantes acervos: RENOTE, RBIE, SBIE e SIMPEP, que estão em destaque no contexto da pesquisa tanto em relação ao foco e escopo, quanto pela alta qualidade de seus trabalhos. Tal revisão contemplou mais de 15,5 mil artigos publicados no cenário nacional.

Apesar da grande quantidade de trabalhos existentes nos acervos analisados, existe uma lacuna em relação à educação básica, em que abordagens específicas podem 
ser consideradas para uma introdução a problemas computacionais. Pouco além, para os Ensinos Técnico e Superior, os trabalhos retratam o uso de OA como uma extensão de disciplinas e enriquecimento dos conteúdos lecionados. Entretanto, é importante que seu uso contribua no processo criacionista da aprendizagem, inclusive com Pensamento Computacional transmitido como formação básica do cidadão, preparando-o para suas vidas e o enfrentamento de problemas do cotidiano, conforme prevê a LDB (LDB, 2021; SYSLO, KWIATKOWSKA, 2014).

De maneira objetiva e conclusiva, acredita-se que a composição dos temas PC e OA seja uma importante chave para novas pesquisas, que sanem as lacunas identificadas e fortaleçam a divulgação do conhecimento. Assim, como trabalhos futuros, pretende-se propor diferentes maneiras de abordar alguns problemas da Teoria da Computação por meio de OA. Inicialmente as pesquisas serão realizadas na busca por ferramentas e jogos que, apesar de não terem como objetivo principal o caráter educacional almejado, podem ser utilizados para explicar conceitos importantes da temática computabilidade.

Com base na revisão da literatura relatada e as lacunas identificadas surge a seguinte questão: quais seriam as práticas embasadas pelo $P C$ e quais os $O A$ podem ser utilizados para abordar tópicos da Teoria da Computação? Embora existam abordagens complexas, desde iniciativas lúdicas a softwares sofisticados e inclusive robótica, é importante destacar que o uso de materiais de fácil acesso e de baixo custo contribuem, fortemente, com a democratização do conhecimento. Nesses casos, para estimular e facilitar a reutilização do conteúdo didático é importante que instrumentos de apoio, como tutoriais e manuais de instruções, sejam disponibilizados em repositórios digitais.

\section{Referências}

Brackmann, C.B. (2017). Desenvolvimento do Pensamento Computacional através de Atividades Desplugadas na Educação Básica. Tese de Doutorado, Centro Interdisciplinar de Novas Tecnologias na Educação da Universidade Federal do Rio Grande Do Sul.

Braga, J.; Menezes, L. (2014). Introdução aos Objetos de Aprendizagem. In: BRAGA, Juliana Cristina (Org). Objetos de Aprendizagem volume 1: Introdução e Fundamentos. Santo André: Editora da UFABC.

Bombasar, J.R.; Santiago, R.; Miranda, E.M.; Raabe, A.L.A (2015). Ferramentas para o Ensino-Aprendizagem do Pensamento Computacional: onde está Alan Turing? In XXVI Simpósio Brasileiro de Informática na Educação.

Carvalho, J.M.; Magalhães Netto, J.F.; Almeida, T.O. (2017). Revisão Sistemática de Literatura sobre o Pensamento Computacional por Meio de Objetos de Aprendizagem. In XXVIII Simpósio Brasileiro de Informática na Educação.

Cordenonsi, A.Z.; Muller, F.M.; Bastos, F.P. (2006). O Ensino de Heurísticas e Metaheurísticas na área de Pesquisa Operacional sob a ótica da Educação Dialógica Problematizadora. RENOTE - Revista Novas Tecnologias na Educação, v. 3, n. 1.

CSUNPLUGGED (2021) - Computer Science without a Computer. Disponível em: http://csunplugged.org, acesso em 29/10/2021. 
Easterbrook, S. (2014). From Computational Thinking to Systems Thinking: A conceptual toolkit for sustainability computing. Proceedings of the 2nd international conference ICT for Sustainability.

Galafassi, F.P.; Gluz, J.C.; Galafassi, C. (2014). Análise Crítica das Pesquisas Recentes sobre as Tecnologias de Objetos de Aprendizagem e Ambientes Virtuais de Aprendizagem. Revista Brasileira de Informática na Educação, v.21, n.3.

Hamming, R.W. (2012). Methods of Mathematics applied to calculus, probability, and statistics. Mineola, New York: Dover Publications, Inc..

IEEE (2002). Draft Standard for Learning Object Metadata (IEEE 1484.12.1), Institute of Electrical and Electronic Engineers (IEEE) Learning Technology Standards Committee (LTSC).

LDB (2021) - Diretrizes e Bases da Educação Nacional. Portal da Presidência da República Planalto. Disponível em http://www.planalto.gov.br/ccivil_03/leis/19394.htm. Acesso: 29/10/2021.

Polya, G. (1945). How to Solve it?. Princeton, NJ: Princeton University Press.

Royal Society. (2012). Shut down or restart: The way forward for computing in UK schools. Disponível: https://royalsociety.org/ /media/education/computing-inschools/2012-01-12-computing-in-schools.pdf, acesso: 29/10/2021.

Santos, C.; Camponogara, M.; Bernardon, D.P. (2016). A inclusão de uma nova ferramenta para o ensino operacional de redes de distribuição de energia elétrica. RENOTE - Revista Novas Tecnologias na Educação, v. 14, n. 2.

Semaan, S.G; Brito, J.A.M.; Coelho, I.M.; Silva, E.F.; Fadel, A.C.; Ochi, L.S.; Maculan, N. A Brief History of Heuristics: from Bounded Rationality to Intractability. IEEE Latin America Transactions, v.19, n.11.

Silva, P.F. (2017). O uso das tecnologias digitais com crianças de 7 meses a 7 anos: Há mudanças na gênese do pensamento operatório de crianças que começam a interagir com as tecnologias digitais na primeira infância? Tese de Doutorado. Universidade Federal do Rio Grande do Sul.

Sipser, M. (2005). Introdução à teoria da computação. Ed.1, Cengage Learning.

Syslo, M.M.; Kwiatkowska, A.B. (2014). Learning Mathematics supported by Computational Thinking. Constructionism and Creativity, pp.258-268.

Wing, J. M. (2006). Computational thinking. Communications of the ACM, v. 49, n. 3.

Zanetti, H.A.P.; Borges, M.A.F.; Ricarte, I.L.M. (2016). Pensamento Computacional no ensino de Programação: uma revisão sistemática da literatura brasileira. In XXVII Simpósio Brasileiro de Informática na Educação. 